\section{Normal laparoscopic anatomy of the caprine pelvic cavity}

\author{
Mujeeb ur Rehman Fazili,1 \\ Riaz Ahmad Shah, ${ }^{2}$ Maajid Hassan Bhat, ${ }^{2}$ \\ Firdous Ahmad Khan, 2 \\ Anubhav Khajuria, ${ }^{3}$ Syed Hilal Yaqoob, 2 \\ Niaz Ahmad Naykoo,2 \\ Nazir Ahmad Ganai2 \\ 1 Division of Veterinary Clinical Service \\ Complex, 2Division of Animal \\ Biotechnology, ${ }^{3}$ Divisions of Veterinary \\ Surgery and Radiology, Faculty of \\ Veterinary Sciences and Animal \\ Husbandry, Sher-e-Kashmir University of \\ Agricultural Sciences and Technology of \\ Kashmir, Srinagar, India
}

\section{Abstract}

Due to the several advantages over conventional procedures, the laparoscopic disease diagnosis and surgery has now started receiving attention in small ruminants. The normal laparoscopic anatomy needs to be described for comparison with the findings in animals with various diseases. The objective of the present study was therefore to describe the laparoscopic anatomy of the caprine pelvic cavity. Adult Bakerwal and Pashmina goats $(n=25)$ of both the sexes were included in this laparoscopy study. All the animals were restrained in dorsal recumbency and Trendelenburg position under lumbosacral epidural anesthesia and sedation. After creating the pneumoperitoneum, the primary port for 5 $\mathrm{mm}$ laparoscope was placed at linea alba $(3.0 \mathrm{~cm}$ cranial to mammary glands in does), and at right paramedian $(3.0 \mathrm{~cm}$ cranial to the rudimentary teat in the bucks) site. Secondary port was placed under direct laparoscopic observation 5-6 cm away from the primary port in horizontal plane, to allow insertion of the grasping forceps. Scan was performed first at the primary port and subsequently through the secondary port for orientation and exploration of the pelvic cavity. The ventral laparoscopic approach provided satisfactory exposure of the pelvic cavity in goats. Comprehensive description of the pelvic organs could be obtained. However, dorsal aspect of the urinary bladder neck and accessory genital organs of male animals could not be visualized. Major complications were not encountered during or after laparoscopy. Laparoscopy a minimally invasive procedure has several advantages over alternate methods of understanding anatomy, physiology and pathology of most of the intraperitoneal pelvic structures in goats. The technique has high pedagogic value. The procedure is safe in experienced hands.

\section{Introduction}

Laparoscopy (keyhole or minimally invasive surgery) is a type of surgical procedure that allows a surgeon with the use of an instrument (laparoscope) inserted transabdominally to access the inside of the abdomen and pelvis without having to make large incisions in the skin. ${ }^{1}$ This modality has diagnostic, therapeutic and prognostic applications. Laparoscopy has many advantages over laparotomy. They include reduced tissue trauma, postoperative adhesions and infections, fast recovery, stimulation of the immunity, better cardiovascular stability and lower pain scores. ${ }^{1-5}$

In recent years, laparoscopy has gained acceptance in veterinary medicine. ${ }^{4}$ In small ruminants, it has been recognized as one of the most promising tools for improvement of the reproductive efficiency, ${ }^{6-8}$ disease diagnosis and treatment. ${ }^{9-11}$ In goats particularly those maintained for dairy purpose or as pets, this state of the art technology is expected to be more popular in the near future.

Laparoscopic anatomy of the abdominal cavity in goats has recently been described.12 That study included female goats only. Additionally, organs located in the abdominal cavity received major consideration. With this background in mind, the present study was aimed to provide description of the laparoscopic anatomy of the pelvic cavity, in goats of both the sexes. The description will be useful for those interested in augmenting animal production as well as for those involved in disease diagnosis, treatment and prognosis of the pelvic disorders in goats. A significant pedagogic value would be an additional attribute.

\section{Materials and Methods}

The prospective study was carried out on twenty apparently healthy, adult, nonpregnant female and five male Pashmina and Bakerwal goats (weighing 25-31 kg and aged 1.5-2.5 years) maintained by the Division of Animal Biotechnology, Faculty of Veterinary Sciences and Animal Husbandry, India. The study was undertaken after receiving due approved from the institutional animal ethics committee. Two days prior to laparoscopy, wide ventral abdominal area from the mammary glands/rudimentary teats up to the umbilicus was clipped and shaved in all the animals. The food and water was withheld for 36 and 24 hours respectively. Regional lumbosacral epidural anesthesia was achieved using 2\% lignocaine hydrochloride $(4.0 \mathrm{mg} / \mathrm{kg})$ plus xylazine hydrochloride $(0.05$ $\mathrm{mg} / \mathrm{kg}$ ). Immediately after satisfactory induction, every goat was shifted to a cradle, placed in dorsal recumbency with all the legs tied
Correspondence: Mujeeb ur Rehman Fazili, House No. 78, Lane 6, Green Park, HIG Colony, Bemina, Srinagar, Kashmir, 190018 India.

Tel.: + 91.941.9095830.

E-mail: fazili_mr@yahoo.co.in

Key words: Goat, laparoscopy, pelvic cavity, pneumoperitoneum, Trendelenburg.

Acknowledgements: financial assistance from NAIP project of Indian Council of Agricultural Research (ICAR) with title "A value chain on zona free cloned embryos for quality animal production from elite buffaloes and Pashmina goats" undertaken at Centre of Animal Biotechnology, SKUAST-K, Srinagar, J \& K, India is gratefully acknowledged.

Contributions: MRF performed all the surgeries and prepared the manuscript; RAS assisted in surgeries; MHB, assisted in surgeries, photography; FAK, photography, postoperative care of the animals; AK, induction of anesthesia, its maintenance and periodic evaluation; SHY, preparation of the animals, maintenance of the optical system; NAN assisted in preparation of the manuscript, maintenance of the pneumoperitoneum; NAG, availability of the animals and drugs, critical evaluation of the manuscript.

Conflict of interest: the authors declare no potential conflict of interest.

Received for publication: 14 May 2015.

Accepted for publication: 17 June 2015.

This work is licensed under a Creative Commons Attribution NonCommercial 3.0 License (CC BYNC 3.0).

(C) Copyright M. ur Rehman Fazili et al., 2015

Licensee PAGEPress srl, Italy

Veterinary Science Development 2015; 5:6012 doi:10.4081/vsd.2015.6012

apart. The abdominal area was subjected to aseptic surgical preparation and properly draped. The cradle was then tilted to approximately $45^{\circ}$ angle with head down (Trendelenburg) position of the animal. Laparoscopic equipment and instruments of Karl Storz, DmbH, Germany were used. Hopkins II straight forward $\left(0^{\circ}\right.$ viewing angle of vision) telescope having $5.0 \mathrm{~mm}$ diameter and $29.0 \mathrm{~cm}$ length, was used. One cm longitudinal skin incision at linea alba, $3.0 \mathrm{~cm}$ cranial to the mammary glands in does and $3.0 \mathrm{~cm}$ cranial to the rudimentary teat in the left paramedian area in the bucks was followed by development of pneumoperitoneum with the use of a Veress needle. After withdrawing the pneumoperitoneum needle, $6.0 \mathrm{~mm}$ trocar-cannula with pyramid tip was passed into the peritoneal cavity at its site (followed by removal of the trocar) to create the primary port. The 
laparoscope was subsequently passed through the cannula into the peritoneal cavity. Systemic examination of the entire pelvic cavity was done by video camera connected to the eye piece of the laparoscope and the images were transferred through the control unit to the television monitor and recorded on a video tape. Secondary port was developed using a 6.0 $\mathrm{mm}$ threaded cannula unit in the right paramedian area (5.0 to $6.0 \mathrm{~cm}$ lateral to the first port). This port was used to pass the instruments for grasping and/or manipulation of the viscera. The laparoscope and the grasping forceps were then exchanged to visualize through the secondary port and manipulate through the primary port.

After completion of the visceral observation session, the accessory instrument and the laparoscope were retracted. The cannulas were removed only after evacuation of the abdominal air. The portal sites were closed with one subcuticular stitch using No 1 chromic catgut. Antiseptic was sprayed over the incision sites.

The animals were shifted from the cradle, placed in sternal recumbency on a level surface and allowed to regain complete motor power before leading them to their sheds. During this period, they were given a dose of amoxicillin-dicloxacillin ( $0.5 \mathrm{~g})$ and meloxicam $(0.5 \mathrm{mg} / \mathrm{kg})$ intramuscularly. Antiseptic dressing of the portal sites was continued daily up to three days following suture removal on day 8 . The animals were watched for complications if any for 10 days following laparoscopy.

Laparoscopy was repeated in does $(n=15)$ after a variable period of two to five months while they were subjected to embryo transfer.

\section{Results}

Lumbosacral injection of lignocaine plus xylazine produced satisfactory analgesia of the abdominal area (up to the umbilical region) along with general sedation in all the goats. Supplementation or additional anesthetic administration was not required in these animals.

One of the animals that had not been fasted properly, showed regurgitation of the rumenal contents towards the end of the session. The animal however did not develop any complications later.

Preoperative fasting was found highly beneficial to reduce the size and motility of the gastrointestinal tract. It not only allowed satisfactory visualization but also prevented inadvertent puncture of the viscera in all the animals.

Pneumoperitoneum resulted in creation of sufficient space within the abdominal wall and in between the viscera; essential for satisfactory visualization and manipulation. Trendelenburg position encouraged abdominal organs to slide cranially exposing whole of the pelvic cavity. Occasionally, some of the animals had to be slightly rotated in the cradle to maintain perfect dorsal recumbency.

Insertion of the laparoscope at primary portal site allowed optimum visualization of the pelvic viscera in animals of both the sexes. By orienting the laparoscope in different direc- tions and planes, various urogenital organs, segments of small and large intestines, ligaments supporting pelvic viscera, major blood vessels, internal inguinal ring and the muscles lining the pelvic cavity were identified.

Urinary bladder as a hollow, thin walled, ovoid organ having bluish semitransparent coloration and tortuous serosal vessels was

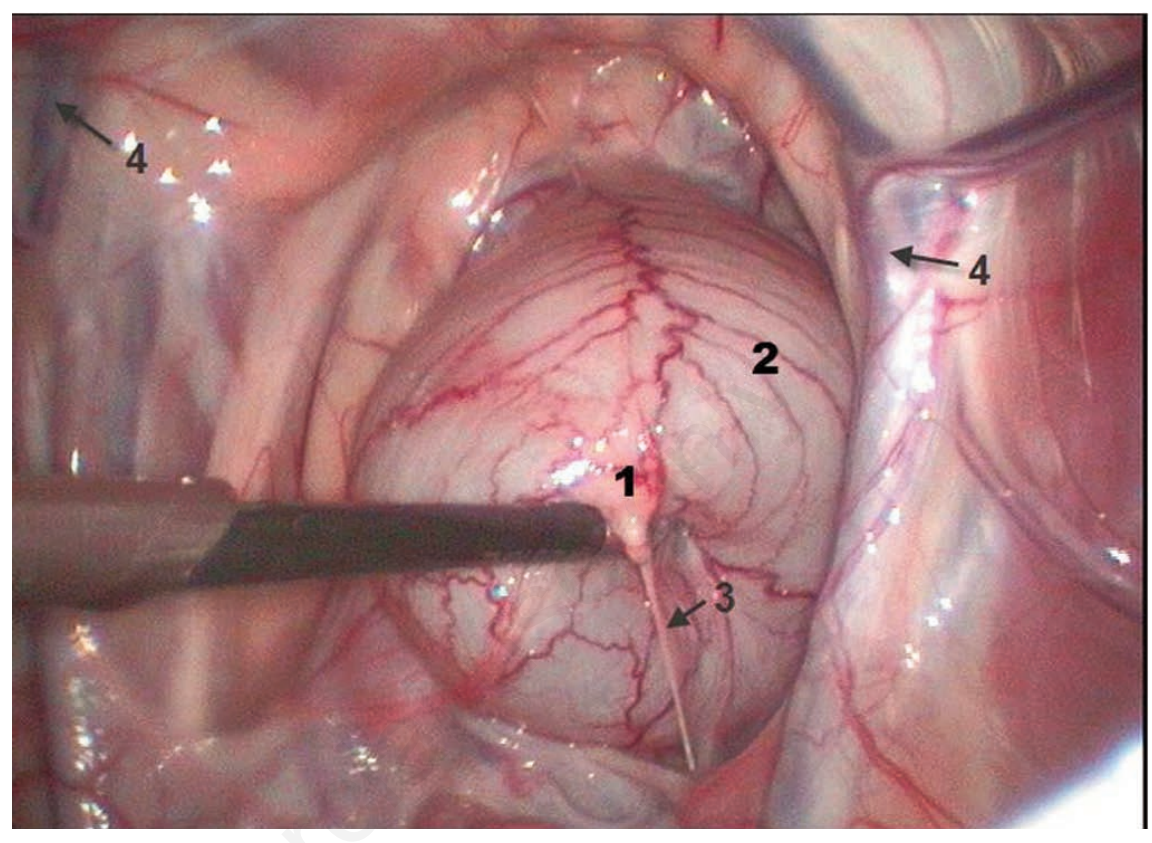

Figure 1. Photograph showing laparoscopic view of pelvic organs and structures in a goat: 1 . remnant of urachus, 2 . urinary bladder, 3 . round liament of bladder, 4 . internal iliac artery.

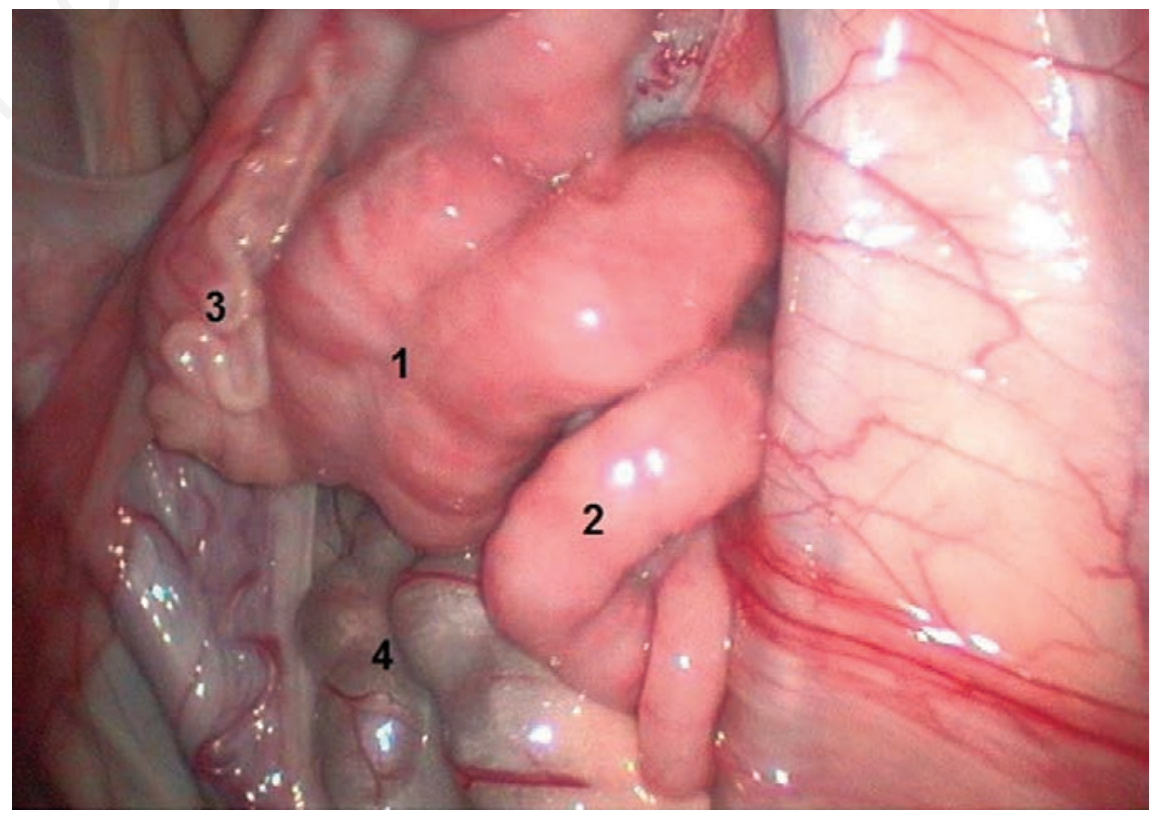

Figure 2. Photograph showing laparoscopic view of pelvic organs and structures in a goat: 1. uterine body, 2. uterine horn, 3. Fallopion tube, 4. distal colon. 
easily located in the central area (Figure 1).

Most of the occasions, it was moderately filled with urine. Vestigial remnant of fetal urachus was visible at the vertex of the bladder. The round and lateral ligaments were detected (extending from both the sides of the bladder towards the corresponding lateral wall of the pelvic cavity) after elevation of the bladder by the accessory instrument (Figure 1). The elevation of the bladder from its vertex area towards the ventral pelvic wall was possible only in animals with minimal residual urine. The lateral ligaments prevented visualization of the dorsal neck area of the urinary bladder in animals of both the sexes and the accessory genital organs in male animals. In does the uterus (Figure 2) and broad ligaments and in bucks the rectum was located dorsally.

Cecum as a curved organ was located to its full length after it was pulled out of the supraomental recess or the later pushed cranially. Cecum appeared as a dilated, oval viscous of larger diameter than the small intestines showing caudally directed blind end (Figure 3 ). Its wall thickness was more but diameter lesser than that of the urinary bladder.

The descending colon was identified by its segmentation into the pellets and its fecal contents. The fecal pellets appeared darker bead like round objects within its lumen (Figure 2).

Small intestines were identified due to their mesenteric attachment and emanating vasculature, peristalsis, thin wall, smooth surface, smaller diameter and more closely coiled pattern than the colon and the cecum (Figure 3 ).

Segment of the uterine horns as pinkish, tubular, soft tissue, curved, smooth structures without peristalsis and without prominent superficial vasculature were visible just cranial to the bladder only in goats with mild to moderate distension of urinary bladder (Figure 2). The ovaries, their proper ligaments and mesovarium, fallopian tubes, broad ligaments and uterine horns were inspected in detail only after retraction of the overlying gastrointestinal structures, grasping and elevation of the reproductive tract by accessory instruments (Figure 2). Body of the uterus and rectogenital pouch was viewed easily after elevation of the uterus against the ventral abdominal wall by the grasping forceps. The fallopian tubes appeared tortuous with light pinkish color starting from the narrow tip of each uterine horn (Figure 2). The tubes were best exposed when the mesovarium was displaced or spread by accessory instrument. Surfaces of the ovaries were best viewed after the mesovarian ligaments were picked-up. The ovaries appeared as oval organs (Figure 4) and located craniolateral to the fimbria of the fallopian tube. Laparoscopy provided better understanding of the position of supporting structures of the uterus and ovaries. The ovaries were sus- pended in the abdominal cavity by proper and mesovarian ligaments. The mesovarium appeared as the cranial portion of the broad ligament suspending ovaries with the lateral abdominal wall and through which blood vessels, lymphatics and nerves passed to the ovary. The cyclic morphological alterations were detected on the ovaries, including ovarian follicle and corpus hemorrhagicum, corpus

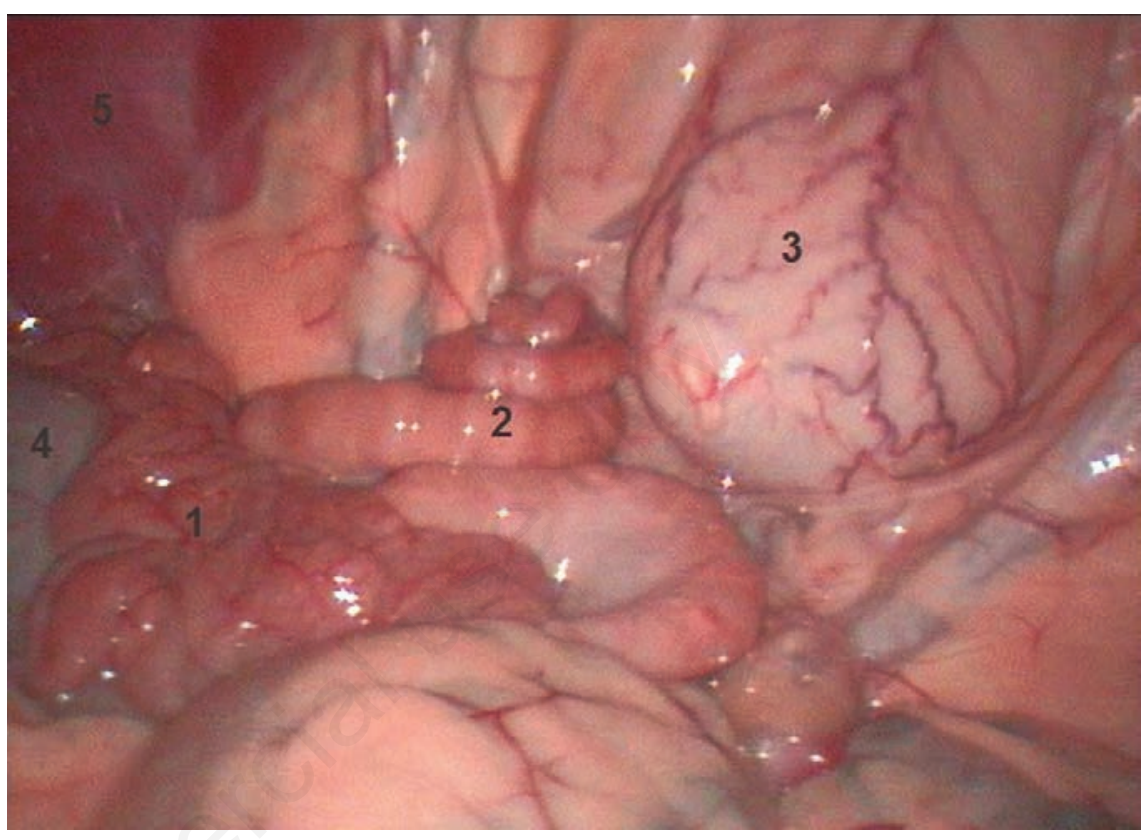

Figure 3. Photograph showing laparoscopic view of pelvic organs and structures in a goat: 1. small intestine, 2. uterine horn, 3. urinary bladder, 4. caecum, 5. internal abdominal obliques muscle.

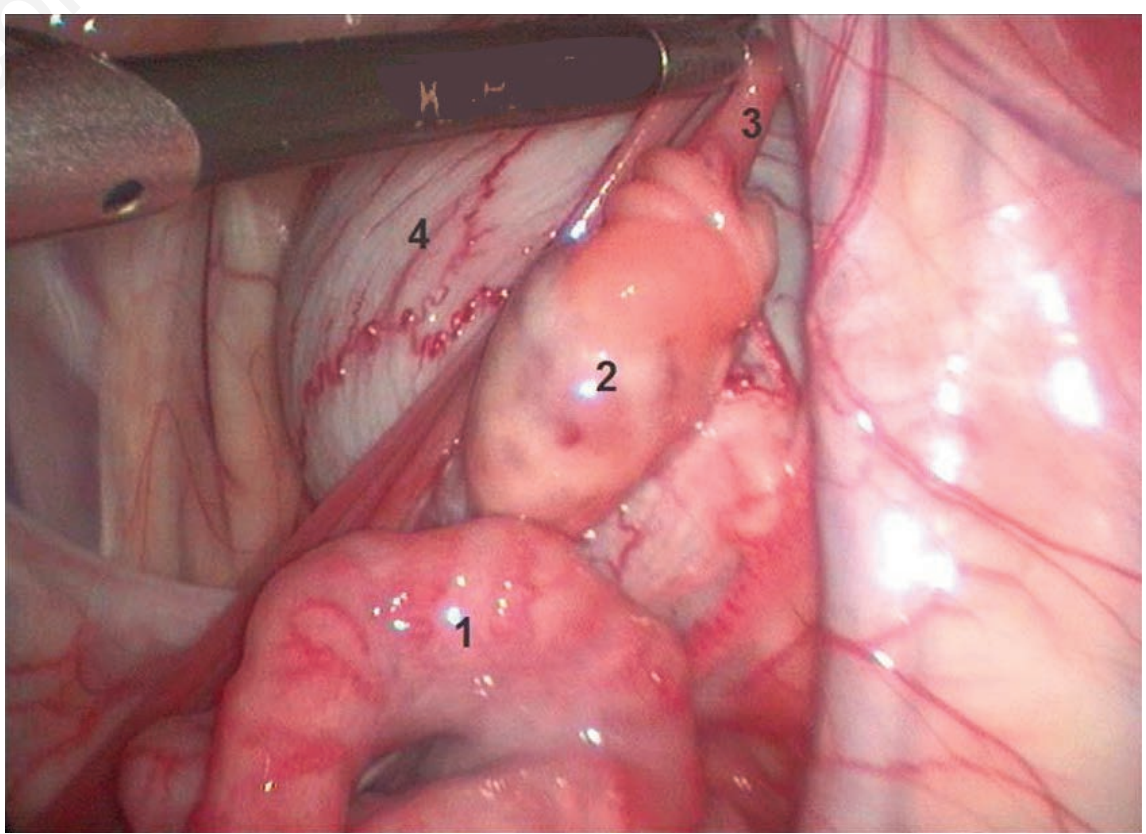

Figure 4. Photograph showing laparoscopic view of pelvic organs and structures in a goat: 1. uterine horn, 2 . ovary, 3. mesovarium ligament, 4. urinary bladder. luteum and corpus albicans. in the lateral pelvic wall and the structures passing through it were visualized bilaterally (Figure 5). In male animals, the vas deferns was easily located as white cord like tubular structure passing from each internal inguinal ring towards the neck of the bladder (Figure 5).
Internal inguinal ring as a slit like aperture 


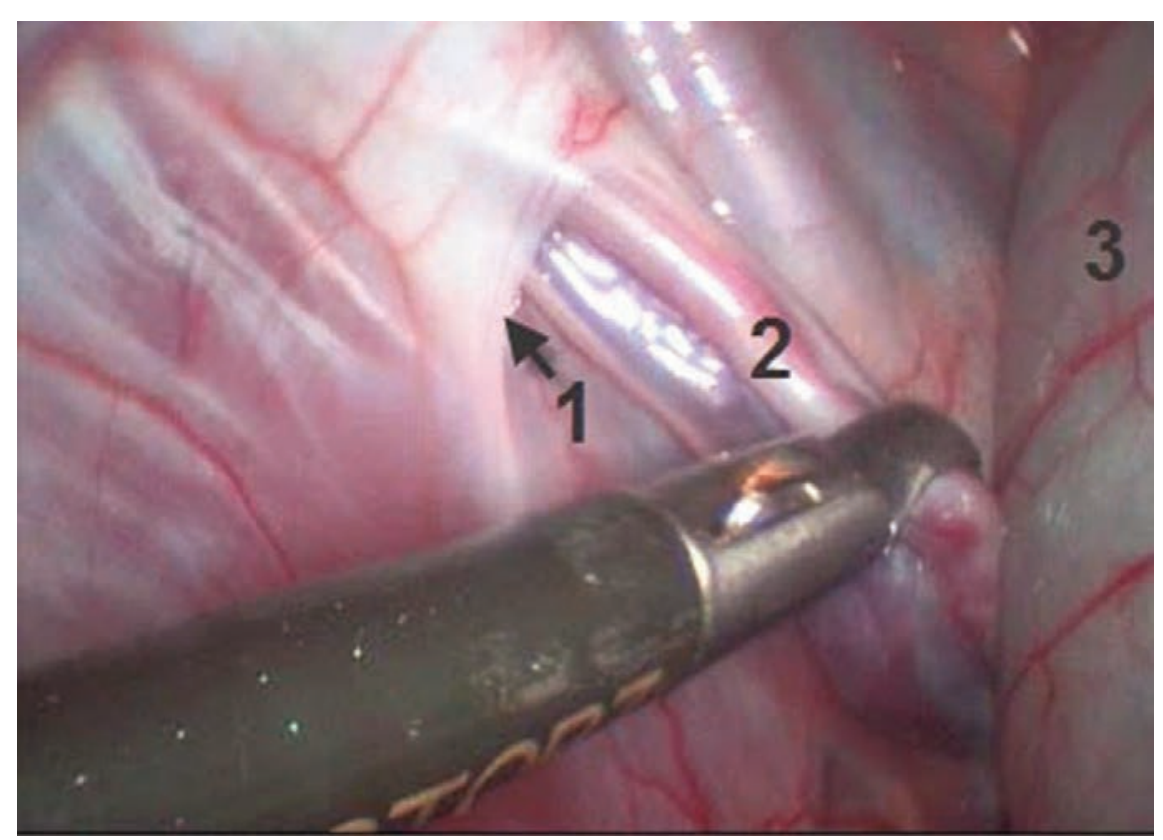

Figure 5. Photograph showing laparoscopic view of pelvic organs and structures in a male goat: 1 . inguinal ring, 2 . vas deferns, 3 . urinary bladder.

The internal iliac artery and its bifurcation into cranial and caudal gluteal arteries were visible bilaterally near the caudal end of the pelvis (Figure 1).

All of the goats recovered normally within two hours from induction of the regional anesthesia. However mild subcutaneous emphysema around the portal sites in two goats subsided subsequently within three and four days respectively. On follow up laparoscopy, neither adhesions nor any other complication that could be attributed to the previous procedure was detected in the pelvic cavity of any animals.

\section{Discussion and Conclusions}

Laparoscopy offers the advantage of direct observation of internal anatomy of the abdominal and pelvic cavities. In addition to the several diagnostic and therapeutic advantages, the technique has particularly a very important pedagogic value. Magnification of the images and the organs being separated from each other (due to pneumoperitoneum), this technique helps in identification of even the smaller structures, rings, apertures and openings not seen in standard celiotomy approach. ${ }^{12}$

Laparoscopy in small ruminants has been performed either under general anesthesia or infiltration of the portal sites with the local anesthetic agent along with sedation.6,8-12 However, we used lumbosacral epidural anesthesia satisfactorily without complications. In small ruminants, this technique has several advantages. Unlike local infiltration of the portal sites, lumbosacral epidural anesthesia results in abdominal wall relaxation which is particularly beneficial during laparoscopy.

In the present study, usage of $5.0 \mathrm{~mm}$ laparoscope provided good panoramic and close-up view of the pelvic cavity. Usage of $0^{0}$ laparoscope allows satisfactory orientation and easier manipulation of the instruments. ${ }^{13-15}$ It also maximizes light transmission compared with laparoscopes with an offset viewing angle. ${ }^{1}$ Usage of cold light fountain xenon, with 175 Watt lamp and $4.5 \mathrm{~mm}$ fiber optic light cable provided satisfactory illumination for videoscopy. However, several workers have recommended 300 -W xenon light source to perform laparoscopy. 1,15,16

Preoperative fasting was mandatory before any laparoscopic procedure. ${ }^{17-19}$ Fasting for 36 hours but not 24 hours was found sufficient to decrease the content of rumen and large intestine and reduce intestinal peristaltic motility in the goats included in this study. It not only reduced the risk of organ penetration during Veress needle introduction or undue pressure of the cranially displaced abdominal organs on diaphragm but also improved observation of abdominal/pelvic structures. In most of the animals, preoperative fasting is essential when a ventral surgical approach is used. ${ }^{20}$

In male animals the mid ventral area being occupied by penis and prepuce made it necessary for us to introduce the primary port at paramedian location. This however did not interfere in locating and visualizing different pelvic organs and structures. In the dogs and cats, insertion of the Veress needle caudolater- al to the umbilicus and directed towards the pelvis to avoid falciform ligament and injury to the spleen has been reported. ${ }^{21}$

Trendelenburg position encourages abdominal organs to slide cranially, exposing caudal field. $1,20,22$

Laparoscopy provided a comprehensive description of the normal laparoscopic anatomy of the caprine pelvis in dorsal recumbent position.

The subcutaneous emphysema noticed in two goats resolved without intervention. This finding corroborates well with an earlier report. ${ }^{23}$ Follow up in the female goats indicated that the exploratory laparoscopic procedure is safe. Repeated laparoscopy does not increase the risk of intra-abdominal complications. ${ }^{24}$

The minimal exposure of the abdominal/pelvic cavities to outside atmosphere and the least visceral handling was done during laparoscopy. Therefore, the systemic antibiotic and analgesic were administered only once postoperatively in all the animals. Such drugs are reportedly required only for 24 hours following laparoscopy in cattle. ${ }^{1}$

From this study, it is concluded that laparoscopy a minimally invasive procedure has several advantages over alternate methods of understanding anatomy, physiology and pathology of most of the intraperitoneal pelvic structures in goats. The procedure is also safe in experienced hands.

\section{References}

1. Bouré L. General principles of laparoscopy. Vet Clin North Am Food Anim Pract 2005;21:227-49.

2. Buunen M, Gholghesaei M, Veldkamp R, et al. Stress response to laparoscopic surgery. Surg Endosc 2004;18:1022-8.

3. Babkine M, Desrochers A. Laparoscopic surgery in adult cattle. Vet Clin North Am Food Anim Pract 2005;21:251-79.

4. Yanmaz LE, Okumus Z, Dogan E. Laparoscopic surgery in veterinary medicine. Vet Res 2007;1:23-39.

5. Newman KD, Harvey D, Roy J. Minimally invasive field abomasopexy techniques for correction and fixation of left displacement of the abomasum in dairy cows. Vet Clin North Am Food Anim Pract 2008;24: 359-82.

6. Shin ST, Jang SK, Yang HS, et al. Laparoscopy vs. laparotomy for embryo transfer to produce transgenic goats (Capra hircus). J Vet Sci 2008;9:103-7.

7. Silva JCB, Okabe WK, Traldi AS. From cattle to sheep: a view of the difficulties and success of commercial in vitro production of sheep embryos. Anim Reprod 2012;9: 
195-200.

8. Toni D, Plamen T, Vladimir $\mathrm{P}$, et al. Laparoscopy-promising tool for improvement of reproductive efficiency of small ruminants. Mac Vet Rev 2013;35:5-11.

9. Franz S, Dadak AM, Schoffmann G, et al. Laparoscopic-assisted cystotomy: an experimental study in male sheep. Vet Med Czech 2009;54:367-73.

10. Zhang J, Wang H, Liu Y, et al. Laparoscopic splenectomy in goats. Vet Surg 2009;38: 406-10.

11. Hunter BG, Huber MJ, Riddick TL. Laparoscopic-assisted urinary bladder marsupialization in a goat that developed recurrent urethral obstruction following perineal urethrostomy. J Am Vet Med Assoc 2012;241:778-81.

12. Kassem MM, El-Gendy SAA, Abdel-Wahed RE, El-Kammar M. Laparoscopic anatomy of caprine abdomen and laparoscopic liver biopsy. Res Vet Sci 2011;90:9-15.

13. Prescott R. Optical principles of laparoscopy. In: Harrison R, Wildt D, eds.
Animal laparoscopy. Baltimore: Williams and Wilkins; 1980. pp 15-29.

14. Freeman LG. Operating room set up, equipment, and instrumentation. In: Freeman LG, ed. Veterinary endosurgery. St. Louis: Mosby; 1999. pp 3-23.

15. Chamness CJ. Non disposable instrumentation for equine laparoscopy. In: Fisher AT, ed. Equine diagnostic and surgical laparoscopy. Philadelphia: W.B. Saunders Company; 2002. pp 37-49.

16. Magne ML. Laparoscopy: instrumentation and technique. In: Tams TR, ed. Small animal endoscopy, 7th ed. St. Louis: Mosby; 1999. pp 367-375.

17. Seeger KH, Klatt PR. Laparoscopy in the sheep and goat. In: Harrison RM, Wildt DE eds. Animal Laparoscopy, Chapter 6, 1980; Williams and Wilkins; Baltimore, London.

18. Maxwell D, Kraemer D. Laparoscopy in cattle. In: Harrison RM, Wildt DE eds. Animal Laparoscopy, 1980; Williams and Wilkins; Baltimore, pp 133-157.

19. Steiner A, Zulauf M. Diagnostic laparoscopy in the cow. Schweizer Archiv Fur Tierheilkunde 1999;141:397-406.

20. Kolata RJ, Freeman LJ. Access, port placement and basic endosurgical skills. In: Freeman Lynetta J ed. Veterinary endosurgery. St. Louis: Mosby; 1999. pp 39-60.

21. Kenawy AA. Experimental laparoscopy and instrumentation in dogs. Assiut Vet Med J 1998;39:51-62.

22. Vilos GA, Ternamian A, Dempster J, Laberge PY. Laparoscopic entry: a review of techniques, technologies, and complications. J Obstet Gynecol Can 2007;29:43365.

23. Anderson DE, Gaughan EM, St-Jean G. Normal laparoscopic anatomy of the bovine abdomen. Am J Vet Res 1993;54: 1170-6.

24. Naoi M, Kokue E, Takahashi Y, Kido Y. Laparoscopic-assisted serial biopsy of the bovine kidney. Am J Vet Res 1985;46:699702. 